\title{
Spacewatch Astrometry and Photometry of Near-Earth Objects
}

\author{
Robert S. McMillan ${ }^{1}$, Jeffrey A. Larsen ${ }^{2}$, Terrence H. Bressi ${ }^{3}$, James \\ V. Scotti ${ }^{3}$, Ronald A. Mastaler ${ }^{3}$ and Andrew F. Tubbiolo ${ }^{3}$ \\ ${ }^{1}$ Lunar and Planetary Laboratory \& Steward Observatory, \\ University of Arizona, \\ Kuiper Space Sciences Building \#92, \\ Tucson, AZ 85721, U. S. A. \\ email: bob@lpl.arizona.edu \\ ${ }^{2}$ Physics Dept., U. S. Naval Academy, \\ Mail Stop 9C, \\ Annapolis, MD 21402, U. S. A. \\ email: larsen@usna.edu \\ ${ }^{3}$ Lunar and Planetary Laboratory, op. cit.
}

\begin{abstract}
The Spacewatch Project uses four telescopes of apertures 0.9-m, 1.8-m, 2.3-m, and 4-m on Kitt Peak mountain in Arizona for followup astrometry of priority NEOs. Objects as faint as $V=23$ on the MPC's NEO Confirmation Page, targets of radar, potential impactors, targets of spacecraft observations or visits, and PHAs with future close approaches to Earth receive priority for astrometry.
\end{abstract}

Keywords. minor planets, asteroids, astrometry, atlases, surveys

The Spacewatch Project is the longest-running of all present programs of astrometry of solar system objects and the first to use charge-coupled device (CCD) imaging detectors for such work (Gehrels et al. 1986, McMillan et al. 1986, 2007, 2010, 2012, 2014, Larsen et al. 2014, Scotti et al. 2014). Nowadays, to fill an underrepresented niche, Spacewatch specializes in followup observations of Near-Earth Objects (NEOs) of high priority while they are faint. Many asteroids and comets are being lost owing to insufficient followup astrometry, but only the most important ones can be followed with the limited resources available. We produce an annual average of $\sim 8500$ lines of astrometry of $\sim 1,000$ different NEOs. We contribute to the removal of half of the objects that were retired from impact risk lists. Our observations at elongations as small as 46 degrees support followup of hazardous NEOs and NEOs discovered by the NEOWISE spacecraft (Wright et al. 2010, Mainzer et al. 2011a,b), and we lead the community of NEO followup astrometrists in numbers of early observations of the latter type of objects (Nugent et al. 2015). Per year we observe about 35 radar targets, 50 NEOs that were measured by NEOWISE, and 100 potential rendezvous destinations. In the last 3 years we have observed half of all NEOs and PHAs observed by anyone in that time. We lead in followup of provisionally designated PHAs while faint $(\mathrm{V} \geqslant 22)$; contributing $41 \%$ of all such observations.

With the Steward Observatory 0.9-meter telescope on Kitt Peak, site code 691, we survey with a $1.85 \times 1.71 \mathrm{deg}$ mosaic of CCDs near opposition and at low elongation in the east. Three-pass coverage is 1400 square deg per lunation with an image scale of 1 arcsec per pixel and a $\mathrm{R}$ mag limit $\sim 21$. Astrometry of asteroids is good to \pm 0.3 arcsec. Twelve years of such uniform surveying, amounting to 17 terabytes of data, will go live on the web in 2015 to support incidental astrometry \& precoveries of NEOs (Larsen et al. 2015). Beyond the solar system, the cadence of the Spacewatch mosaic 
data will provide sampling of variable stars and galaxies on time scales from tens of minutes to 12 years, a range rarely available from databases of this type.

On the Spacewatch 1.8-m telescope, site code 291, the field of view $=21 \times 21$ arcmin and the pixel scale $=0.6$ arcsec/pixel. The $\mathrm{V}$ mag limit $=23$ and the astrometric residuals on faint NEOs are \pm 0.3 arcsec.

Our output with the Bok 2.3-m telescope of the Steward Observatory and the Mayall 4-m telescope of the Kitt Peak National Observatory from 2010-2015 was 1605 lines of astrometry on 249 different NEOs, including 94 different PHAs. 343 observations were made of PHAs with $\mathrm{V} \geqslant 22$. Our average calendar span extension on large PHAs using the Bok and Mayall telescopes is 6 months, twice as long as that of the next most effective observing station. We extend the span of calendar time coverage on PHAs an average of 3.8 times. For 38 of 72 PHAs we added another observed opposition. With the Bok and Mayall we reduce uncertainties of orbital elements an average of a factor of 6 and the uncertainty of the time of perihelion passage an average of a factor of 19 (G. Williams 2014 private communication).

\section{Acknowledgements}

We gratefully acknowledge NASA's NEO Observation Program, The IAU's Minor Planet Center, JPL's NEOWISE Team led by A. K. Mainzer, JPL's NEO Office, Steward Observatory, the U. S. Naval Academy, the estates of R. L. Waland and R. S. Vail, and other private donors. This work is also based in part on observations at Kitt Peak National Observatory, National Optical Astronomy Observatory, which is operated by the Association of Universities for Research in Astronomy (AURA) under cooperative agreement with the National Science Foundation.

\section{References}

Gehrels, T., et al. 1986. AJ 91, 1242-1243: http: //adsabs .harvard.edu/abs/1986AJ . . . .91.1242G .

Larsen, J. A., et al. 2014. AAS/DPS mtg \#46, \#213.12: http: //adsabs.harvard.edu/abs/2014DPS . . .4621312L .

Larsen, J. A., et al. 2015. AAS/DPS mtg \#47, http: //adsabs . harvard. edu/abs/2015DPS . . . 4730814L.

Mainzer, A., et al., 2011a, ApJ 731, Issue 1, article id. 53: http: //adsabs.harvard . edu/abs/2011ApJ . . 731 . .53M .

Mainzer, A. K., et al., 2011b, ApJ, 743, Issue 2, article id. 156, http://adsabs . harvard . edu/abs/2011ApJ . . 743 . 156M .

McMillan, R. S., et al. 1986. In Instrumentation in Astronomy: Proc. S.P.I.E. 627 (D. L. Crawford, Ed.), VI, 141-154: http://adsabs.harvard.edu/abs/1986SPIE. 627 . 141M .

McMillan, R. S., et al. 2007. In Proc. IAU Symp. 236: Near Earth Objects, our Celestial Neighbors - Opportunity and Risk, A. Milani, G. Valsecchi, and D. Vokrouhlicky, Eds. (Cambridge U. Press), pp 329-340: http: //adsabs .harvard.edu/abs/2007IAUS . 236. .329M .

McMillan, R. S., et al. 2010. AAS/DPS mtg \#42, \#13.22; BAAS 42, 1057 : http: //adsabs.harvard.edu/abs/2010DPS . . .42.1322M .

McMillan, R. S., et al. 2012. AAS/DPS mtg \#44, \#210.14: http: //adsabs.harvard.edu/abs/2012DPS . . .4421014M and

McMillan, R. S., et al. 2014. AAS/DPS Mtg \#46, \#414.11: http: //adsabs.harvard.edu/abs/2014DPS . . . 4641411M .

Nugent, C. R., et al. 2015. ApJ, 814117 http: //adsabs.harvard.edu/abs/2015ApJ . .814 . 117N .

Scotti, J. V. et al. 2014. AAS/DPS Mtg \#46, \#414.17: http: //adsabs.harvard.edu/abs/2014DPS . . .4641417S .

Wright, E. L., et al. 2010. AJ 140, 1868-1881: http: //adsabs.harvard.edu/abs/2010AJ . . .140.1868W . 